\title{
IoT-Based Solid Waste Management Solutions: A Survey
}

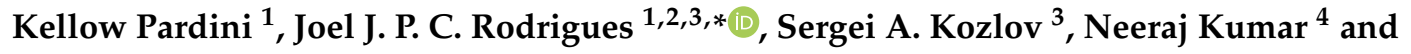 \\ Vasco Furtado ${ }^{5}$ \\ 1 National Institute of Telecommunications (INATEL), Santa Rita do Sapucaí-MG 37540-000, Brazil; \\ kellow.pardini@mtel.inatel.br \\ 2 Covilhã Delegation, Instituto de Telecomunicações, 1049-001 Lisbon, Portugal \\ 3 Photonics and Optoinformatics Department, ITMO University, 197101 St. Petersburg, Russia; \\ kozlov@mail.ifmo.ru \\ 4 Department of Computer Science and Engineering, Thapar University, Patiala 147003, India; \\ neeraj.kumar@thapar.edu \\ 5 PPGIA, University of Fortaleza (UNIFOR), Fortaleza-CE 60811-905, Brazil; vasco@unifor.br \\ * Correspondence: joeljr@ieee.org
}

Received: 27 November 2018; Accepted: 19 December 2018; Published: 1 January 2019

\begin{abstract}
With the increase of population density and the rural exodus to cities, urbanization is assuming extreme proportions and presents a tremendous urban problem related to waste generation. The increase of waste generation has been considered a significant challenge to large urban centers worldwide and represents a critical issue for countries with accelerated population growth in cities. The Internet of Things (IoT) and cloud computing offer an automation possibility through cyberphysical systems that will change the way solid waste management is performed. Considering IoT requirements, a review analysis of waste management models available in the literature is performed in detail in this paper. Then, a deep review is undertaken of the related literature based on IoT infrastructure for efficient handling of waste generated in urban scenarios, focusing on the interaction among concessionaires and waste generators (citizens) from the perspective of a shorter collection time with reduced costs, as well as citizenship promotion. An IoT-based reference model is described, and a comparison analysis of the available solutions is presented, with the goal to highlight the most relevant approaches and identify open research issues on the topic.
\end{abstract}

Keywords: Internet of Things; smart cities; smart waste bin; solid waste management; waste disposal management

\section{Introduction}

Waste management is a name given to a waste collection system, including its transportation, disposal or recycling. This term is attributed to waste material that is produced through a human activity which must be handled to avoid its adverse effect for health and for the environment. Most often, waste is managed to reuse available resources. Waste management methods may differ between developed countries, between an urban and a rural environment, or between an industrial and a residential area. The management of waste in metropolitan and rural areas is the general responsibility of a municipality, while waste produced by industries is their responsibility and managed by themselves.

According to data released by the United Nations Department of Economic and Social Affairs, the share of the urban population worldwide is expected to reach $66 \%$ by 2050 , compared to $52 \%$ in 2014, resulting in increased waste production in cities. Data released by the World Bank Group 
confirm that waste generation rates are growing. In 2012, cities worldwide generated about 1.3 billion tons of solid waste, representing 1.2 kilograms of waste generated per person-day. With rapid population growth occurring along with urbanization, urban waste generation is projected to rise to 2.2 billion tons by 2025, confirming that municipal solid waste (MSW), the main by-product of an urban lifestyle, is growing even faster than the rate of urbanization. This increase in municipal and industrial waste generation, together with stricter regulations aimed at ending illegal waste disposal, stimulate the growth of applications for better waste management. Other factors that have driven the growth of applications designed for the effective management of waste worldwide are directly linked to the constant use of recycling techniques, the cycle of technological innovation, the application of advanced techniques for waste collection, and the use of technologies based on IoT and big data. There is also a vision of strengthening waste management based on public initiatives aimed at building more correct and safer environments, as well as reducing greenhouse gas emissions.

According to Allied Market Research, Portland, Oregon [1], waste management worldwide is expected to grow at an annual rate of $6.2 \%$ by 2023, with greater growth in the emerging Asia Pacific region. In Europe, this sector grew by more than 30\% in 2016 and growth is expected to continue to accelerate due to the presence of advanced infrastructure and the high demand of several interested sectors.

Currently, there are increasing initiatives by governmental and public authorities in relation to waste management to efficiently improve the collection and intelligent disposal of waste generated by a city. These are already considering the accelerated pace of urbanization worldwide and the expansion of the industrial sector, and the manufacturing and healthcare industries that are likely to produce a significant amount of waste and can already be efficiently treated by smart management. Moreover, growth of infrastructure facilities and a rising adoption of advanced waste management systems in developing economies with the goal of using cost-effective and waste-time disposal methods should positively impact the growth of smart management of waste.

The great precursor of technological development that has led to innovations in the waste management sector is undoubtedly the advance of the Internet [2]. The Internet has revolutionized the world and offers global connectivity. Similarly, the Internet of Things (IoT) [3] is also set to underpin significant change and represents an Internet evolution known as the next generation of the Internet (i.e., the Fourth Industrial Revolution).

The IoT began with the increasing number of interconnected physical objects providing interactions. The IoT paradigm [4] has a main role as a key facilitator of the integration of various application solutions and communication technologies, such as identification and tracking $[5,6]$, sensor networks, wired and wireless actuators [7], improved communication protocols, and distributed intelligence for objects. According to the Internet Business Solutions Group (IBSG), a milestone of IoT emergence occurred when the Earth's population was exceeded by the number of objects connected to the Internet, which happened in 2008-2009. IBSG predicts that by 2020, about 50 billion devices will be connected to the Internet [8-11]. IoT can include a large number of applications designed to assist in many sectors, such as industry, transportation, markets, education, agriculture, healthcare, environment, and smart cities (Figure 1) [12-15].

The European Union has defined smart cities (SC) as a system where people interact and use energy, materials, services, and waste to stimulate economic development and improve the quality of life. These interaction flows are considered intelligent because they make strategic use of infrastructures, services, information, and communication in planning urban management, a way to meet the social and economic needs of society. Despite being a relatively recent concept, the smart city topic has already become synonymous with sustainable development within global discussions on sustainability [16]. Currently, cities in emerging countries are investing heavily in smart products and services to sustain economic growth and, at the same time, developed countries need to upgrade existing urban infrastructures to remain competitive. Among the evolutions that have been taking place to classify an 
intelligent city, a vast range of applications can be listed, such as waste management based on an IoT approach [17-21].

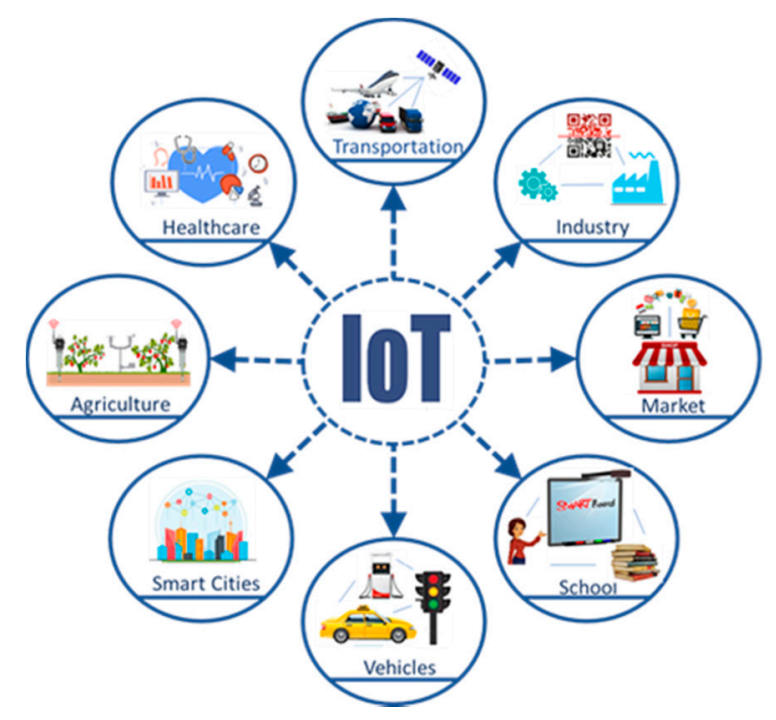

Figure 1. Illustration of an Internet of Things (IoT) general framework with several vertical applications.

The IoT concept predicts a world in which physical, digital, and virtual objects are interconnected in a network supporting higher order applications. The intelligence of objects comes from the automated data processing of an existing state or the environmental state in which it is immersed. These data are then transmitted to a processing node where they are analyzed, and an appropriate performance profile is determined, considering data acquired from various objects. This actuation profile is then transmitted back to the smart object [22]. The waste management system is included in this context because it has a large number of containers with an inconsistent level of filling that can last from days to weeks, with the possibility of seasonal changes, as well as different emptying requirements, such as distances and type of waste. However, biomedical, chemical, and electronic residues have specific collection points, usually with uniform production and long filling periods.

There is considerable difficulty in identifying the filling level of the dumps because of the differences in the waste packaging process, such as the variety and the irregularity of the discarded materials, which in a certain way generate unnecessary costs to the municipal collection system [23,24]. Recognizing the vast scope that IoT offers for waste management in urban environments, it is the focus of this study. This survey analyzes the state-of-the-art based on intelligent waste management through IoT. Thus, the main contributions of this paper are the following:

- $\quad$ An in-depth review of state-of-the-art on solid waste management;

- Presentation of architecture models for solid waste management identified in the literature based on IoT requirements;

- Comparison of the most promising solutions and identification of open research issues able to suggest further research works on the topic.

The remainder of this paper is organized as follows. Section 2 overviews the types and methods of solid waste disposal and Section 3 provides an insight regarding solid waste management. Section 4 addresses available IoT architecture reference models for waste management systems. Section 5 presents the value chain of IoT-based waste management systems and Section 6 describes the most relevant standard protocols used in waste management systems. Discussion and open issues are present in Section 7. Lessons learned are pointed out in Section 8, and, finally, Section 9 concludes the paper. 


\section{Types and Methods of Waste Disposal}

The garbage generated by various segments of society can be classified according to its composition (physical characteristics) and destination. This classification is fundamental because it facilitates the selective collection, the recycling, and the definition of the most appropriate goal. These solid wastes discarded by urban municipalities represent a highly heterogeneous volume of matter, as well as a more homogeneous load of industrial and hospital waste [25]. Currently, a selective collection is the basis for proper waste management and the primary method adopted around the world when the goal is recycling. For a waste management system based on IoT, it is vital that classification is done previously, so specific containers for each waste type should be considered.

As an example, the collection of solid waste, in London, is done according to the requirements of selective collection. It uses garbage bags or different colored containers, such as toxic waste in red; hospital waste in yellow; hospital waste after disinfection in blue; black household waste; glass bottles are divided into green, black, brown groups, according to their types and colors, and placed in different containers [26]. Then, the different types of considered waste are described as follows:

- Organic Waste. It is the garbage derived from organic waste [27]. They are generated mainly in residences, restaurants, and commercial establishments that work with food. They must be separated from other types of waste since they are mostly destined to municipal landfills.

- Recyclable Waste. It is all the waste that can be used in the process of transformation to other elements or in the manufacture of raw materials [28]. It is generated in residences, companies, and industries, and must be separated so that the selective collection teams gather and then deliver to final processing in cooperatives and recycling companies.

- Industrial Waste. They are the residues, mainly solid, originating in the process of production at industries. It is usually composed by leftovers of raw materials destined for recycling or reuse in the industrial process [29].

- Hospital Waste. It is the waste originated in hospitals and medical clinics and can present contamination and transmit diseases to people that come into contact with it [30]. It should be treated according to established standards, with all possible care. This type of waste is intended for companies specializing in the treatment of such waste, where it is usually incinerated.

- Commercial Waste. It is the one produced by commercial establishments, such as clothing stores, toys, and appliances. This waste is almost entirely for recycling [31].

- Green Waste. It is the material that results, mainly, from the pruning of trees, branches, trunks, barks, and leaves that fall in the streets. Because it is organic matter, it could be used for composting and production of organic fertilizer [32].

- Electronic Waste. This is the waste generated by the disposal of consumer electronics products that no longer work or have become obsolete [33]. For disposal, there are appropriate places, such as companies and cooperatives that operate in the area of recycling. They send this waste in a way that does not cause damage to the environment.

- Nuclear Waste. It is the one that is generated, mainly, by nuclear plants. It is a highly dangerous waste because it is a radioactive element and should be treated according to strict safety standards [34].

\section{Solid Waste Management}

Several published papers cover different aspects of IoT technology for waste management solutions. For example, in Reference [35], the authors present a solution that, through intelligent monitoring, allows the planning of garbage collection. Through a Smart-M3 platform (extension of cross-domain search for triple-based information), it is possible to interoperate applications from different domains of information and communication, and to bring a great ease of implementation. The solution is developed in two phases: the first is a monitoring phase where the levels of waste inside the compartments are constantly measured, transmitted and stored; the second is a phase 
in which the calculation of the collected information is applied to optimize the waste collection routes. In Reference [36], the authors address a dynamic waste management model through a set of infrastructure services for Smart Cities based on IoT. With the use of sensors, radiofrequency (RFID) and actuators in the process of monitoring identification, this set is divided into three phases: (i) Planning and execution of waste collection using solutions of routing in trucks with dynamic adaptation of routes according to restrictions introduced; (ii) transport to a specific place according to the type of waste; and (iii) recycling of waste that can be reused. However, it was basically used in the first one that deals with the planning and collection of waste. The dynamic term presents the capacity of the system to adapt in real time to the parameters and the plans that interest the collection of residues during the activity. These works provide a macro view of a waste management infrastructure with emphasis on the processes applied on excellence management of waste without entering the merit of the sensors applied for monitoring and the used communication methods.

The authors of [37] provide a solution identified as cloud-based smart waste management (Cloud SWAM). It addresses a solution with specific containers for each type of waste (organic, plastic, bottles, and metal) equipped with sensors that constantly monitor and update their status to the cloud, where stakeholders are connected to receive information relevant to their interest. The system acts not only in waste management but also in the decision of the best collection route, tracing a more economical path within the metropole. Moreover, in Reference [38], a new management model that specifically focuses on the discovery of better areas for the construction of landfills is introduced. As landfills are used as the final destination of commercial and industrial residential waste, identifying an appropriate location within large urban areas requires special attention, because there should be concern about the economy, the environment, and public health. The solution uses the information collected by the waste management system associated to a language that uses a genetic algorithm that assists in the selection of suitable land for the construction of landfills. In Reference [39], the authors describe the various methods of waste disposal in which waste management can be applied. They feature an integrated solution of fill level sensors with solar-powered waste compaction called a smart box, which optimizes waste collection. The information is transferred to a server in the cloud via wireless communication and is applicable to any type and size of container, and stakeholders can log in to the server and access the data in real time through monitoring of the smart boxes. In these addressed solutions, the authors present a more targeted approach to the concessionaires, taking into account the reduction of collection costs, providing information about the waste of interest, and lower transportation costs for disposal in landfills to the real problem faced by large companies. Cities refer to poor management of too much waste generated. Additionally, the proposed architectures are not well portrayed.

Intelligent waste management as a model for the improvement of waste collection is presented in Reference [40]. In some countries, such as Australia, municipalities usually charge fees for waste produced in the city and generally measure the weight of garbage for each neighborhood or street and then rate the average of each user per household. This collection model is not the most accurate, and as the cost of waste disposal increases every year, waste producers (users of the system) demand a solution that reduces the cost and changes the form of charging, applied through a fixed rate. Intelligent waste management can solve this issue by ensuring that the user is taxed only based on the waste that produce. In addition, the system can reduce costs with a large number of containers that are missing or stolen. The work presented here addresses a good proposal when the lack of waste collection affects only the budget of citizens, but the architecture used was not well portrayed.

A review of the literature on the optimization of waste collection and vehicle routing is presented in Reference [41]. To conclude the idea, a multirestricted and multicompartmental routing problem is proposed that, through modeling using container scheduling strategies in a decision making process, produces results that indicate that the differentiated collection has the potential to search the best routing strategies with lower collection costs, which ensures that advances in optimization strategies can present intelligent and ecological solutions. In addition, the study presented in Reference [42] proposes an intelligent system of trash recycling. Preseparation of waste before disposal is required 
where the glass will be destined in brown containers, paper and aluminum cans in blue containers, and plastic products in orange containers. The system automatically evaluates the type and amount of waste disposed of and a benefit is provided in the form of points credited to a card. The accumulated points can be exchanged for an item or even withdrawn through the banking network, simulating a virtual currency. The above authors follow a baseline of specific containers for a preseparation of waste, where a first model focuses on gains provided by a differentiated collection and the second case focuses on the offer of a prize for citizens who discard their waste correctly, but they do not detail how the sensors are not trapped in the solution.

In Reference [43], a solution is proposed to the problem of dumps that are not cleaned in time and reach overflow. The system offers a monitor that triggers an alarm and informs the authorized persons that the container is ready to fill, associated with a screening system through near infrared spectroscopy (NIR) that identifies five types of plastic resin and the remainder of biodegradable waste is destined for the production of biogas. The authors of [44] provide a model for collecting information on the use of garbage and helping dumpers to identify and decide if a particular area needs extra dumps or remove them to other places where they are needed. From the daily trash information, cleaning operators can plan better when they should send their cleaning contingents to empty the boxes and can also set routes for their cleaning trucks. In Reference [45], a "smart bin" solution is provided, where bins are distributed on the streets with a unique ID. Sometimes, when the container is about to be filled, there will be a query in a database to determine who is responsible for that compartment and a global system for mobile communications (GSM) notification will be sent containing the container ID and location. The presented models cover the perspective of intelligent dumps, but in all these models, the sensor component is not very clear, as just a communication model is described.

The authors of [46] present the disadvantages of existing systems in comparison to the method proposed by them. The proposal is based on an Arduino IDE and an 8051 microcontroller that reads the data from an infrared sensor used to measure the depth of waste inside the container, processes and transmits via wireless communication to a central system based on a microcontroller Intel Galileo. The proposal presented by these authors approaches the sensors used in the solution very well and the used communication as well. They have another model based on a better collection route.

A literature review for smart waste management and a comparison of the different methodologies is surveyed in Reference [47]. It focuses on IoT, considering its elements (identification, sensing, communication, computation, semantics, and services) and to its characteristics (anything communicates, anything identifies, and anything interacts).

An algorithm for decision making during waste collection is presented in Reference [48]. Some algorithm models are compared, considering several performance metrics, such as receiving data in motion, multiobjective, loss of data during transmission, and increased data reception. It concludes that not all algorithms that are capable of receiving data in motion also serve the multi-objective or are still able to cope with increased data reception, but none of them can address data loss. Based on this information, some case studies are proposed and some objectives, such as reducing pollution through collection on time, reduce the operational cost, using trucks of appropriate size for demand, and using a better collection route, are considered. The algorithm should also take into account the speed and volume of data input as well as data generated by similar sensors. Thus, an algorithm model to optimize the collection decisions is proposed in this work. Moreover, in Reference [49], the proposed model uses information received from the compartments to define effective routes for each truck during collection. Within the model, some constraints are considered as maximum bin capacity and a Poisson distribution models the waste arrival rate. When a dump reaches a filling threshold, a garbage collection alarm is triggered and sent to the base station which, in turn, communicates with the cloud to process the data and find the best collection route. In the end, the trucks head to the emptying area where the amount of visited dumps is verified and establish the optimal path of collection with the objective of optimization based on lower cost for the waste collection. The solution presents a waste management approach based on three heuristics, considering the nearest vehicle 
first, a collection based on the upper limit, and a collection based on the upper and lower threshold. MATLAB was used to solve the optimization models based on cost and delay, and a Java-based simulator for heuristic methods. The proposals presented by the authors specifically deal with search algorithms for an optimal path for waste collection with a focus on reducing the collection time and cost. In Reference [50], a municipal waste management system for domestic use is presented that focuses on the application of biological and physicochemical methodologies that can eliminate or significantly reduce the stage of waste collection and transportation.

The summary of the comparative assessment of the most promising solutions performed in this survey is presented in Section 7. A total of fifteen research efforts were reviewed and their strengths and weaknesses are analyzed, and each model is classified to represent essential parts of the proposed systems.

\section{Available IoT Architecture Reference Models for Waste Management Systems}

To standardize this IoT segment or vertical, being supported by a reference architecture model is very important, so in the future, these waste management devices, which are called objects in IoT, can be connected (and the interoperability challenge is solved).

The Internet is supported by the Transmission Control Protocol/Internet Protocol (TCP/IP) architecture so that communication between network hosts is possible as it is known today. Similarly, an architecture for IoT-based applications is also necessary, always addressing factors such as scalability, interoperability, reliability, Quality of Service (QoS), etc. According to the author of [51], there are several models and reference architectures available for IoT. Each group or company describes its own, which often causes conflicts of ideas and makes the task of standardization more complex.

Among the predominant reference models, it is possible to mention some initiatives, such as RAMI 4.0, a reference architecture for intelligent factories applied to IoT standards, which began in Germany and later became relevant through the direction of companies from large industrial sectors. Another initiative was launched by a consortium formed by AT\&T, Cisco, General Electric, International Business Machines Corporation (IBM) and Intel, called Industrial Internet Reference Architecture (IIRA), and provided a reference architecture that involves broad discussions and considerations, while Internet of Things Architecture (IoT-A) is an initiative that stimulates an architecture model which covers detailed system requirements [52].

Many project models focus on a typical architecture based on needs analysis or on some layers that form a basic model of a reference architecture. Figure 2 illustrates the basic layered architecture. The most basic approach only considers a three-layer architecture composed by application, network, and perception layers [10]. There are also, in recent literature, some other models that add more abstraction to an IoT architecture, such as the Service Oriented Architecture (SOA) model, the middleware model [51,53,54], and the five-tier model $[11,55,56]$.

Even with a flexible architecture, there are still relevant challenges, especially related to security and privacy. Therefore, to overmatch these challenges, new standard architectures need to be proposed with a focus on critical factors, such as QoS, sustainability, data integrity, confidentiality, and reliability. Next, there is a brief discussion about these layers that, in turn, alternate between the presented models.

- Perception Layer. The IoT architecture perception layer is similar to the physical layer of the Open Systems Interconnection (OSI) model, because it is based on the hardware level and has the responsibility of collecting physical information, processing it, and transferring it to the upper layers through secure channels. It applies technologies for the detection of parameters of physical characteristics through specific sensors, such as weight, temperature, humidity, etc., in addition to the collection of object identification data, such as Quick Response codes (QR codes) and RFID.

- Network Layer. The network layer is responsible for transferring the measured information in the perception layer to the upper layers, where the processing systems are located. and uses ZigBee, Z-wire, GSM, UMTS, Wi-Fi, Infrared, 6LoWPAN. In addition to the basic assignments, the network layer also performs the cloud computing process and the data management process. 
- Middleware Layer. The middleware layer is a layer of software or even a set of sublayers that work to interconnect components of the IoT that would not be possible to communicate otherwise, that is, an interpreter. In addition to providing concurrency so that the application layer can interact with the layer of perception and ensure effective communication, it plays an important role in the development of new technologies.

- Application Layer. The application layer does not directly contribute to the construction of an IoT architecture, but it is in this layer where the various services are built that interface with users, that is, where the interpretation and availability of the information occurs.

- Business layer. This layer is responsible for managing the entire IoT System, including service-related applications such as providing high-level analysis report of the underlying layers, as well as addressing user privacy. The responsibility of creating graphs and business models can be attributed to this layer.

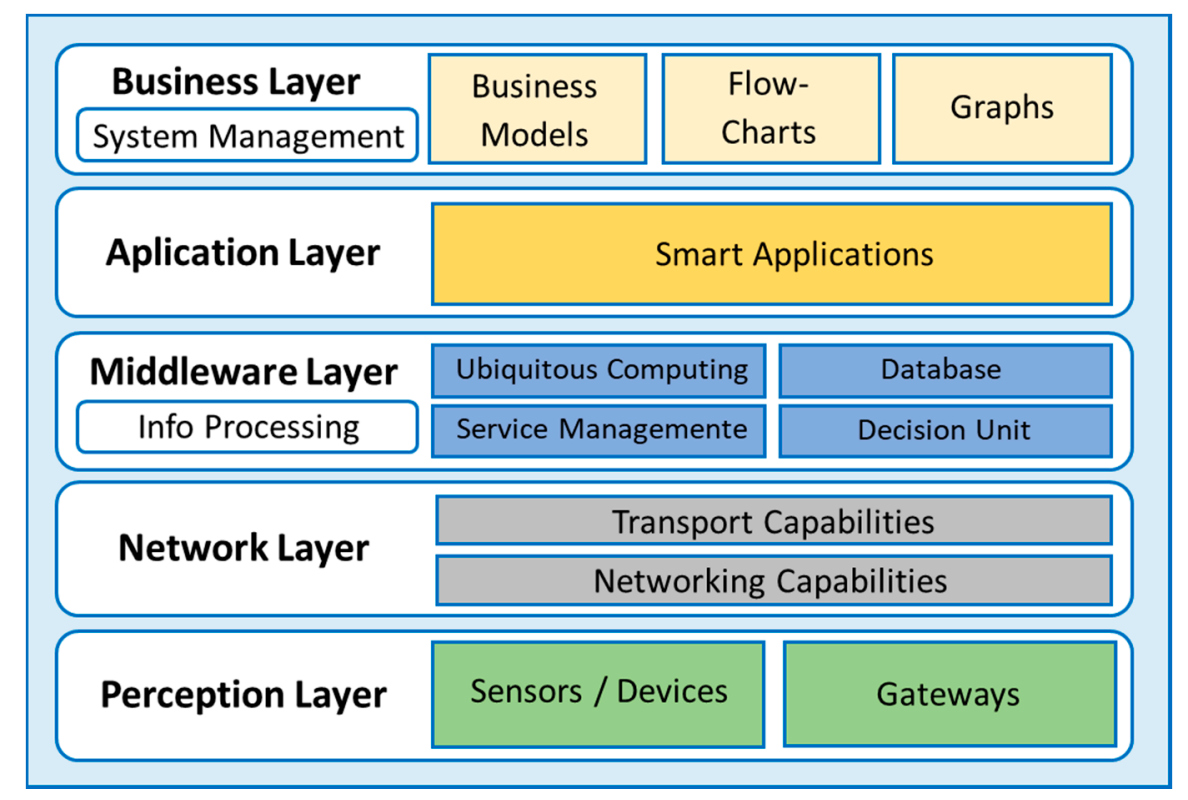

Figure 2. Layered architecture for waste management systems.

Since IoT connects everything to exchange information among themselves, the traffic and the stores within network tend to increase exponentially. Thus, IoT application development depends on technology progress and design following a reference model of an IoT architecture.

\section{Value Chain of IoT-Based Waste Management Systems}

Understanding the integration of several empowering technologies in a system (such as data acquisition and network transmission) offers an improved insight into the meaning of each functionality within the context of IoT [57]. An overview of how these technologies play a key role in an IoT-based solid waste management system is presented in this section. A set of activities is appropriately related from the beginning of its cycle through the identification of each compartment. It is associated with a useful sensing and capable of accurately presenting service level conditions with adequate communication and capable of providing the minimum requirements necessary for the proper functioning of this system. Moreover, an applied computation is added to break the information that meets the users' need within a large amount of collected data accessed by simple services, and it is easy to use until semantic representation of information through an object. Figure 3 illustrates this value chain for IoT-based waste management systems. 


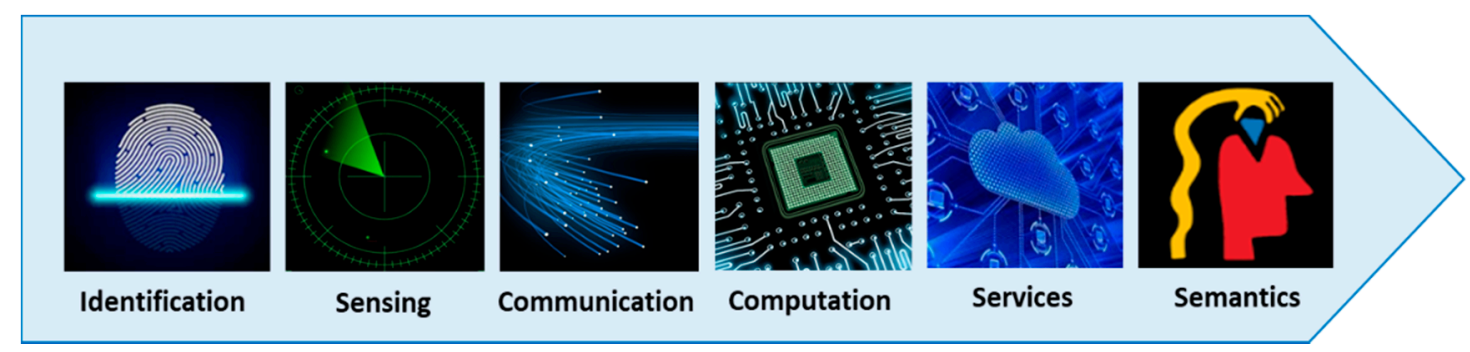

Figure 3. Illustration of a value chain for IoT-based waste management systems.

As may be seen in Figure 3, the proposed value-chain for IoT-based waste management systems considers the following aspects that are described as follows:

- Identification. For IoT, classifying services and linking them to demand is extremely important, so various identification methods are supported by IoT, such as the electronic product code (EPC) and ubiquitous codes (uCode) [58], and GPS trackers [59], which will determine the exact location. The key to identifying a particular object within a telecommunication network is to provide you with an ID and address. The ID refers to the name of the object, e.g., "P1" for a specific pressure sensor and its address refers to a number that identifies that device within the network. The methods of object nesting within an IoT network may include Internet Protocol version 4 and version 6 (IPv4 and IPv6). The IPv6 on low power personal networks (6LoWPAN) [60] provides a compression mechanism over headers. As a method of identifying objects within a network, public IP addressing is used.

- Sensing. Sensing means to capture specific and relevant data from objects on a network and send them to a database or cloud so that they can be analyzed and serve as the basis for decision making in a particular service. Sensors can be classified as intelligent [61], such as actuators [62], or sensitive portable devices. Many IoT solutions associate sensors with single board computers (SBCs) that are devices (for example, Arduino Yun, Raspberry PI, Beagle Bone Black) which connect to application software in a central management to provide information that clients need.

- Communication. In order to integrate different objects and provide specific services within an IoT environment, it is necessary to apply communication technologies such as Wi-Fi [63], Bluetooth [64], Institute of Electrical and Electronic Engineers (IEEE) 802.15.4 [51], LoRa [65], wave Z, GSM/GPRS, broadband code division multiple access (WCDMA), long term evolution (LTE) and Advanced LTE [66,67], near field communication (NFC) [68], Ultra-wideband (UWB) [69] and 6LoWPAN [60,70], and the IoT nodes must operate with low power consumption. RFID [71] is a specific communication technology that can also be considered where a query signal is emitted from the RFID reader against a label called TAG that reflects and returns to the reader. There are different types of TAGs; active TAGs that are battery powered; passive TAGs that operate without the presence of a battery for power supply; and semipassive TAGs that, when necessary, use the board supply [72]. NFC technology operates in a high-frequency band of $13.56 \mathrm{MHz}$ with rates of $424 \mathrm{kbps}$ in a band of a distance of up to $10 \mathrm{~cm}$ [60]. UWB, also known as 802.15.3, is a communications technology designed by the IEEE to operate within areas of low coverage and bandwidth requirements. [69]. Wi-Fi uses radio waves for communication within a range of $100 \mathrm{~m}$ and allows devices to communicate through an ad hoc configuration [63], i.e., without the use of a router. Bluetooth is a communication technology widely used for communication between devices at a short distance. It uses basic radio waves with short wavelengths to guarantee a saving in the consumption of batteries [64]. 802.15.4, developed by the IEEE, provides specifications in low power wireless networks for both the physical layer and the medium access control layer by promoting reliable and scalable communication [73]. Long term evolution (LTE) is a wireless communication standard that enables high speed data transfer between mobile phones based on GSM/UMTS network technologies and encompasses devices in locomotion at high speeds in 
addition to providing multicast-based services and broadcast [66]. LTE Advanced (LTE-A) is an enhanced version of conventional LTE and includes broadband coverage, spatial multiplexing, greater coverage, and better performance with lower latencies [67]. Better known as the fourth generation of mobile communications is an evolution of WCDMA (3G) and GSM/GPSR (2G).

- Computation. It is the unit that represents the computational capacity of IoT, based on software and applications. There is a huge range of hardware development platforms for IoT application operation; some examples are: Arduino [74], UDOO [75], FriendlyARM [76], Intel Galileo [77], Raspberry PI [78], Gadgeteer [79], Beagle Bone [80], Cubieboard [81], Z1 [82], WiSense [83], Mulle [84], and T-Mote Sky [85], but operating systems are seen as vital because they run throughout the entire system execution period. In addition to TinyOS [86], LiteOS [87], and RiotOS [88], which also offer a lightweight operating system, it is possible to cite the Contiki RTOS, widely used in IoT scenarios $[89,90]$ for IoT environments. Cloud platforms are another important computing component within an IoT solution. These platforms provide capabilities for receiving data from intelligent objects to be processed or stored, so that in the future, users can benefit from the knowledge of the extracted data. Data analysis platforms within IoT are crucial due to the specific characteristics of this type of solutions given heterogeneous data and systems integration. Similarly, IoT-based solid waste management works with real time data that require correlation and sharing. To meet these requirements in a system with a large volume of connected devices generating data by different flows, it is increasingly necessary to adopt cloud computing where storage, processing, and connection capacity are needed according to the growing demand for data analysis.

- Services. Within IoT, services can be classified through four classes: Services related to identity that represents the most basic and essential to other services-applications that need to take objects from the real world to the virtual must first identify them; information aggregation services responsible for summarizing the raw information that needs to be processed and exposed to the applications; collaborative-aware services acting on aggregation services in decision making; and ubiquitous services, providing support services.

- Semantics. Semantics refers to the ability to extract knowledge in an intelligent way, but through other possibilities and in the proportion in which the services require [91]. This extraction of knowledge covers the discovery and use of modeling resources and information and includes the recognition and analysis of the data so that it makes sense for the right decision by providing the exact service. Semantics behaves like the brain of IoT, sending demands to the specific resource. Such requirements are supported by semantic web technologies, such as the resource description framework (RDF), OWL (web ontology language), and EXI (efficient XML interchange).

\section{Standard Protocols Used in Waste Management Systems}

IoT requires different protocols to address a full range of activities, such as protocols for sensor data collection, communication protocols, etc. Various working groups, such as the Institute of Electrical and Electronic Engineers (IEEE), the Internet Engineering Task Force (IETF), the World Wide Web Consortium (W3C), EPCglobal, and the European Telecommunications Standards Institute (ETSI) began to include efforts to provide standard support protocols for IoT. For an IoT-based waste management solution, not all of the protocols described below need to be involved, but these represent the main and most used ones within the context of IoT. Their brief description is presented per layer according to their main functionalities: Application, service discovery, and network infrastructure protocols.

\subsection{Application Layer Protocols}

At the application layer, the protocols are used for end-user communication and are usually integrated in middleware solutions for IoT [92]. End-user applications detect systems, which means 
they can communicate directly with lower layers of the protocol stack, such as web servers widely used in system integration and communication between different applications.

- Constrained Application Protocol (COAP). The COAP is an application layer protocol developed to support applications within IoT systems $[93,94]$. Based on the Representational State Transfer (REST) functionalities over HTTP [95], REST is a transport protocol used in networks with low power nodes, mobile applications, and social networks, being able to transfer data between client and server in a more direct way, in addition to being a cached connection protocol. Unlike REST, COAP is linked to User Datagram Protocol (UDP), which makes it a lighter and more appropriate protocol for IoT applications, containing adaptations of HTTP functionalities for low power consumption when operating on links in the presence of noise and packet loss.

- Message Queue Telemetry Transport (MQTT). MQTT [96] is a publishing and signing transport protocol based on a TCP/IP server-client structure developed for the connection between embedded applications and middleware. It uses one-to-one, one-to-many, and many-to-many routing mechanisms, ideal for IoT systems, providing flexibility and simplified deployment. MQTT has a fixed 2-byte header suitable for devices with limited resources, such as connections with low bandwidth, battery leaks or untrusted links, and IoT requirements.

- Extensible Messaging Presence Protocol (XMPP). The XMPP is an instant messaging protocol over the Internet independent of operating system, designed for chat, voice and video calls, and telepresence [97]. It supports authentication, access control, privacy metering, encryption, and interoperates with other protocols. XMPP communication, based on text using XML, establishes an overload to the system that is solved with XML streams compression using EXI [98] discussed and based on Reference [99].

- Advanced Massage Queuing Protocol (AMQP). AMPQ [100] is an open standard IoT connection layer protocol applied to a message-oriented environment with a publishing and signing structure. It supports reliable communication through primitives that guarantee delivery but requires a reliable transport protocol, such as TCP. It is facially interoperable with other protocols with communication-based on message transfers and queues using a SWAP to route messages to the appropriate queues.

- Data Distribution Service (DDS). DDS [101] is a subscription and publishing protocol developed for real time communications of machine to machine (M2M). In contrast to AMPQ and MQTT, DDS has a decentralized structure and does not require the presence of a broker. It uses multicast as a form of guaranteed traffic delivery and excellent QoS that supports 23 queues with a variety of communication parameters, such as security, urgency, priority, durability, and reliability.

\subsection{Service Discovery Protocols}

Due to a large number of devices connected and given the need to ensure the proper functioning of the applications developed for IoT based systems, a resource management mechanism is essential for excellent coverage of the technology. Thus, the system must be able to discover resources and register the services automatically. The most notorious protocols to meet these needs are domain name system (DNS), multicast (mDNS) and DNS Service Discovery (DNS-SD). Current research studies aim to adapt lighter versions to the IoT environment.

- Multicast DNS (mDNS). mDNS is a very flexible protocol and uses the DNS namespace locally, being a timely option for Internet devices because it does not require manual configuration or an administration that manages the device and is capable of operating without an infrastructure or even in failures. The name query is done through multicast messages, in which the client requests all domain nodes the Internet Protocol (IP) address for a specific name. At that moment, all the ones in the network update the caches with the provided address [102].

- DNS Service Discovery (DNS-SD). The DNS-based discovery service (DNS-SD) performs the service delivery function required by clients through $\mathrm{mDNS}$, enabling customers to discover the 
desired services using standard DNS messages. Like mDNS, DNS-SD does not require a naming configuration [103] and the DNS packets are sent through the UDP transport protocol, having as destination a multicast address. A first step in finding the necessary services is to find the corresponding IP address of the respective host, and then the pairing function is sent, also via multicast, containing the essential details for connection as the IP/Port pair of the connected hosts, so that the names of the instances can be kept constant, increasing reliability.

\subsection{Infrastructure Protocols}

Infrastructure protocols provide communication between devices and the network, i.e., they are based on connectivity between different types of systems and devices, which may use different data types and may be spread out over considerable distances. Thus, the Internet is a connecting option between them.

\subsubsection{Routing Protocol}

Routing Protocol for Low Power Lossy Networks (RPL). The RPL [104,105] was developed to support the minimum routing requirements, always considering a robust topology, when the network is faced with environments where the links have great influence of noises or losses of packages. It guarantees the delivery of traffic from the simplest point-to-point to more complex traffic such as point-to-multipoint and multipoint-to-point. The core of RPL is represented by a target-driven acyclic graph (DODAG), where there is only one root and the network nodes have known their parents and establish redundant paths by establishing one as the primary (faster route to increase performance), but has no information on inferior relationships, children. In order to keep the topology in place and with its routing information up to date, RPL uses some types of control messages, such as the DODAG information object (DIO), which is used to determine the distance between each node of the network until the root based on specific metrics to choose the preferred parent path. Another type of message is the destination announcement object (DAO) that provides information about the traffic of receiving data. The third type of message is the DODAG information request (DIS), used by a node to acquire DIO messages from an accessible adjacent node. The last type of message is DAO confirmation (DAO-ACK), which is a response to a DAO message sent from a DAO target to a DAO parent or DODAG root [106].

\subsubsection{Network Adaptation Layer Protocol}

6LowPAN. Wireless personal area networks (WPANs) are characterized by differences with older link layer technologies, such as limited packet size (127 bytes for IEEE 802.15.4). This difference in packet length is required to ensure low bandwidth, necessitating the creation of an adaptation layer that corresponds to the length of the IPv6 header within the IEEE 802.15.4 specifications. This IETF 6LoWPAN working group developed the 6LoWPAN (low power personal network IPv6) standard that specifies the mapping required by IPv6 over WPANs [107] and provides IPv6 header compression to reduce transmission overhead and fragmentation to meet the requirement of the IPv6 maximum transmission unit (MTU) and support routing on networks where delivery will be through multiple jumps. The 6LoWPAN significantly reduces the IPv6 overhead in such a way that a small datagram can be sent through a single 802.15.4 frame packet at best and the IPv6 headers can be compressed in two bytes [108].

\subsubsection{Link Layer Protocol}

IEEE 802.15.4. This protocol specifies the physical layer (PHY) and the medium access control (MAC) sublayer in low rate wireless private area networks (LR-WPAN) [29]. Due to its specifications (low cost, low power, low data rate) it is widely used in IoT, M2M, and WSNs, as well as providing reliable communication with a high level of security, offering encryption and authentication to networks with a large number of IEEE 802.15.4 standard network topologies that may be in a star, peer-to-peer, 
mesh, and tree format. The star topology specifically contains a total function device (FFD) that acts as the master of the PAN network and is located in the center of the topology and is intended to control all other nodes and other reduced function devices (RFD). The topology point to point contains an FFD, and other nodes communicate with each other or through intermediate nodes. Tree topology is a particular case of point-to-point topology and consists of an FFD and common nodes [51].

\subsubsection{Physical Layer Protocols}

Bluetooth Low Energy (BLE). Compared to previous versions, Bluetooth Smart uses a short range radio up to 100 meters, ten times longer than the classic Bluetooth and guarantees a 15-fold slower latency [109]. It operates with low power consumption at a transmission power between $0.01 \mathrm{~mW}$ and $10 \mathrm{~mW}$, which prolongs the useful life of the device for up to years and makes the BLE a suitable suitor for IoT applications [110], more efficient than the ZigBee not only with regard to the consumption of energy as the rate of power transmitted per bit [111]. The BLE allows a star topology with master devices or writes with a discovery mechanism based on sending messages from the slaves to the master through a dedicated advertising channel that are checked by the master for the discovery to complete. Except when two devices are exchanging data, they are in standby mode.

Electronic Product Code (EPC). The EPC is a technology where an individual identification number is stored in an RFID tag used in supply chain management to identify items [112]. The RFID system is divided into two main components, the radio signal transponder (tag) and a tag reader. This tag uses an object's identity storage chip and an antenna to allow communication with the reader utilizing radio waves reflected in the tag so that after, the read information is sent to a specific computer application called object name service (ONS). OEPC is considered a promising technique for the future of IoT applications due to its openness, scalability, interoperability, and reliability [113].

Global System for Mobile Communications/General Packet Radio Services (GSM/GPRS). GSM networks were deployed throughout as an evolution of first-generation mobile communication systems. Initially, they were developed for voice transmission, but later they started to support data transmission through some specific timeslots at a low $9.6 \mathrm{kbit} / \mathrm{s}$ rate in the uplink and downlink. After a technical evolution of the GSM, the HSCSD, the data rate had a rise and went to reach $14.4 \mathrm{kbit} / \mathrm{s}$ in the uplink and $43.2 \mathrm{kbit} / \mathrm{s}$ in the downlink. GPRS was made available to GSM users from GSM version 97 and enabled the use of data services, such as Internet browsing, WAP access, and SMS/MMS, but unlike the previous version, GPRS uses a packet-switched mode and shares the same transmission channel only when the data are to be addressed. The achievable data rates in GPRS depend on the supported multislot class that can reach $21.4 \mathrm{kbit} / \mathrm{s}$ with a maximum of 8 downlink or uplink slots [114].

Wideband Code Division Multiple Access (WCDMA). WCDMA was set to provide high speed, packet-switched data services, enabling the more efficient use of the spectrum which provides higher transmission rates of up to $2 \mathrm{Mbit} / \mathrm{s}$. WCDMA supports access to Internet-based services, such as fixed-line services. However, WCDMA has been defined without second generation backward compatibility requirements [115].

Long Term Evolution-Advanced (LTE-A). LTE-A encompasses a set of communication protocols that are well suited to machine type communication (MTC) infrastructures, machine-to-machine communication that does not require human interference, in addition to IoT, especially for smart cities where the long term durability of the infrastructure is expected [116]. LTE-A outperforms other cellular mobile communication solutions related to the cost and scalability of the service and has an architecture that is divided into two parts where the first, called a control network $(\mathrm{CN})$, contemplates mobile devices and treats the IP packets. The other, a radio access network (RAN), deals with wireless communication via radio and establishes the user plan and control protocols. The RAN uses base stations (evolved node) that are connected by an interface, called X2 since the connection of RAN as $\mathrm{CN}$ is made through an interface, called S1. Mobile devices or MTCs can connect to base stations 
either directly or through the MTCG gateway. They may also have direct communication with other MTC devices.

With the advancement of communication infrastructure to 5G, the current available technologies will be incorporated into this concept, meaning that $5 \mathrm{G}$ guarantees the specific needs as required. For example, some applications require low network latency, others require high bandwidth, and there are others that only require connectivity because of their low volume of data being transmitted, such as IoT-based applications. Considering these requirements for IoT applications and the current legacy networks under use, it is assumed to take over this demand until the NB-IoT and eMTC networks are fully available for the applications to use as a communication infrastructure.

\section{Open Issues and Challenges}

The summary of the comparative evaluation of this study is presented in Tables 1-3, which include research efforts based on waste management using IoT, with particular attention to waste bins, which will receive the dumps and generate information through an IoT infrastructure.

Table 1 deals with the physical infrastructure of waste bins, such as types of waste supported by the container (organic, glass, plastic, paper, metal, toxic, or general waste without any selection criteria); bins positioning (indoor or outdoor); the pneumatic tube that automatically compacts garbage to decrease volume; and recycling and processing points for the refuse to be returned as a raw material or processed for the correct disposal. Through the provided study, each model is classified considering the impotent parts presented in each system. Regarding the physical infrastructure, in two models, waste bins for the organic discard are considered; in five models, waste bins for glass discarding; in six models, waste bins for the disposal of plastics; in five models, waste bins for paper discard; in four models, waste bins for metal disposal; in only one model, waste bins for toxic waste disposal; and in eight models, general waste. In just one model, the dumps can be positioned both externally and underground; in the others, only externally. Pneumatic tubes are incorporated into two models, five models consider recycling points, and processing points for organic waste are supported in only one model.

Table 1. Physical infrastructure comparison considering the most relevant solutions available in the literature.

\begin{tabular}{|c|c|c|c|c|c|}
\hline Ref. & Ins Type & Bins Location & Pneumatic Pipes & Recycling Points & Processing Points \\
\hline [35] & $\begin{array}{l}\text { Glass; Plastic; Paper; } \\
\text { General Waste }\end{array}$ & Outdoor & Disregard & Not Supported & Not Supported \\
\hline [36] & $\begin{array}{l}\text { Organic; Glass; Plastic; } \\
\text { Paper; Metal; Toxic }\end{array}$ & $\begin{array}{c}\text { Outdoor; } \\
\text { Underground }\end{array}$ & Incorporated & Supported & Supported \\
\hline [37] & $\begin{array}{l}\text { Organic; Glass; Plastic; } \\
\text { Paper; Metal }\end{array}$ & Outdoor & Disregard & Supported & Not Supported \\
\hline [38] & General Waste & Outdoor & Disregard & Not Supported & Not Supported \\
\hline [39] & General Waste & Outdoor & Incorporated & Not Supported & Not Supported \\
\hline [40] & General Waste & Outdoor & Disregard & Not Supported & Not Supported \\
\hline [41] & Glass; Plastic; Paper; Metal & Outdoor & Disregard & Supported & Not Supported \\
\hline [42] & Glass; Plastic; Paper; Metal & Outdoor & Disregard & Supported & Not Supported \\
\hline [43] & Plastic & Outdoor & Disregard & Supported & Not Supported \\
\hline [44] & General Waste & Outdoor & Disregard & Not Supported & Not Supported \\
\hline [45] & General Waste & Outdoor & Disregard & Not Supported & Not Supported \\
\hline [46] & General Waste & Outdoor & Disregard & Not Supported & Not Supported \\
\hline [47] & General Waste & Outdoor & Disregard & Not Supported & Not Supported \\
\hline [48] & Not Specified & Not Specified & Not Specified & Not Specified & Not Specified \\
\hline [49] & Not Specified & Not Specified & Not Specified & Not Specified & Not Specified \\
\hline
\end{tabular}

Table 2 deals with IoT technologies considering the following: RFID support; types of used sensors (capacity, weight, temperature, humidity, chemical, pressure); automatic actuators (prevent excessive deposit); cameras positioned to overlap sensor function; global positioning system (GPS); and IoT architecture (solution not mentioned and declared as implicit or solution developed and declared as defined). RFID is embedded in five models, a capacity sensor is present in twelve models, 
and weight in three, while temperature, humidity, chemical, and pressure sensors are present in only one model.

Table 2. IoT Technology comparison for the most relevant solutions available in the literature (Table 1).

\begin{tabular}{ccccccc}
\hline Ref. & RFID & Sensors & Actuators & Camera & GPS & Architecture \\
\hline$[35]$ & Disregard & $\begin{array}{c}\text { Capacity; Weight } \\
\text { Capacity; Weight; }\end{array}$ & Disregard & Disregard & Disregard & Implied \\
[36] & Incorporated & $\begin{array}{c}\text { Temperature; Humidity; } \\
\text { Chemical; Pressure }\end{array}$ & Incorporated & Disregard & Incorporated & Defined \\
{$[37]$} & Disregard & Capacity & Disregard & Disregard & Disregard & Implied \\
{$[38]$} & Disregard & none & Disregard & Disregard & Disregard & Defined \\
{$[39]$} & Disregard & Capacity & Disregard & Disregard & Disregard & Implied \\
{$[40]$} & Incorporated & Capacity & Disregard & Disregard & Disregard & Defined \\
{$[41]$} & Incorporated & Capacity & Disregard & Disregard & Disregard & Defined \\
{$[42]$} & Incorporated & Capacity & Disregard & Disregard & Disregard & Defined \\
{$[43]$} & Disregard & Capacity; Weight & Disregard & Disregard & Incorporated & Defined \\
{$[44]$} & Disregard & Capacity & Disregard & Disregard & Incorporated & Defined \\
{$[45]$} & Disregard & Capacity & Disregard & Disregard & Disregard & Defined \\
{$[46]$} & Disregard & Capacity & Disregard & Disregard & Disregard & Defined \\
{$[47]$} & Incorporated & Capacity & Disregard & Disregard & Disregard \\
{$[48]$} & Not Specified & Not Specified & Not Specified & Not Specified & Not Specified & Not Specified \\
{$[49]$} & Not Specified & Not Specified & Not Specified & Not Specified & Not Specified & Not Specified \\
\hline
\end{tabular}

To conclude, Table 3 deals with software analysis, i.e., how the information will be used. It can follow a dynamic collection planning; a dynamic routing of the collection; and the experimental evaluation for each research effort (Simulator or Real). In the category of software analysis, five models for dynamic programming are considered and the dynamic routing is also present in five models, while real experimental data are adopted just in one model, with all other models using simulators.

Table 3. Experimental analysis comparison for the most relevant solutions available in the literature (Table 1).

\begin{tabular}{lccc}
\hline Ref. & Dynamic Scheduling & Dynamic Routing & Experimental Data \\
\hline$[35]$ & Not defined & Not defined & Simulator \\
{$[36]$} & Defined & Defined & Simulator \\
{$[37]$} & Defined & Defined & Simulator \\
{$[38]$} & Not defined & Not defined & Simulator \\
{$[39]$} & Defined & Defined & Simulator \\
{$[40]$} & Not defined & Not defined & Simulator \\
{$[41]$} & Not defined & Not defined & Simulator \\
{$[42]$} & Not defined & Not defined & Simulator \\
{$[43]$} & Not defined & Not defined & Simulator \\
{$[44]$} & Not defined & Not defined & Real \\
{$[45]$} & Defined & Defined & Simulator \\
{$[46]$} & Defined & Defined & Simulator \\
{$[47]$} & Not defined & Not defined & Simulator \\
{$[48]$} & Defined & Defined & Simulator \\
{$[49]$} & Defined & Defined & Simulator \\
\hline
\end{tabular}

Among all the papers analyzed, from physical infrastructure, many authors propose a waste management model through IoT with collection emphasis, offering scheduling models with dynamic routing for greater effectiveness of collection, using low fuel consumption. Other authors focus on specific bins for each type of waste that promote to users a selective disposal way; there are even models where a bonus based on the amount of recyclable waste is offered to the user and models where the user, based on a mobile app, can query particular green waste points. There are also authors who propose recycling and processing points not relevant to this research. In a general context, all the models have a focus on the collection system. 
Considering aspects of IoT technology, some authors incorporate the identification of a waste bin through RFID and others not, a fact that makes it not necessary when a GPS is considered, but few models adhere to GPS technology. The variety of sensor types is not well explored in most models, which can lead to false positives when waste disposal is low in volume and high in weight. Automatic actuators are not necessary, since not all types of discards are actually compressible. Cameras installed in bins are a fact that can be discarded when using appropriate sensor models. The waste management system has its architecture explicitly defined when dependent on a standard or implicit model when part of the overall design of the system.

One of the critical points of solid waste management that are being studied today is waste collection. This research effort makes a lot of sense when we stop to reflect that $80 \%$ of the costs spent on waste management are used in fleets of trucks that travel daily to cities, collecting waste discarded by the population [117], a situation that expresses a reasonable basis for a collection process to be practiced, with optimized routes where any improvement achieved can represent a considerable saving of time and money.

Many studies aiming to optimize routes for solid waste collection often relate them to the problem of the street vendor [118] (one of the most studied optimization problems), defined as a salesman who needs to visit several cities passing through each of them only once and return to the home city by running the course at the shortest distance. A system to calculate the better collection routes can be modeled as the problem of the hawker, and the solution of this problem can be through exact algorithms that are an algorithm that always finds the ideal solution for an optimization problem or by heuristic algorithms that can sometimes produce worse solutions. This great challenge of solid waste management systems has been an opportunity for study and proposing robust algorithms capable of dealing with adverse issues presented by IoT-based systems. Within these adversities, the main aspects that can be considered include the large volume of data offered, the speed of receiving such data, the data heterogeneity, and the data loss during transmission. Running a data analysis from heterogeneous sources (in the order of Terabytes or even being able to reach Petabytes, which arrive at high speed and often with losses during transmission) becomes hard work that can take a tremendous amount of time and there is not a useful parameter for a solid waste management system or even an unlikely one for some algorithms. Moreover, in Reference [119], the authors propose an algorithm-based tool model with automatic learning "machine learning" that stipulates efficiency metrics to be used to obtain help in making decisions, based on open information.

Among the major algorithms available in the literature, the following are relevant: Backtracking search algorithm [120], ArcGIS [121], heuristic [122], and particle swarm optimization [123]. Recently, a study was published in Reference [48], where a comparison of these algorithms is presented by adding an expectation algorithm model to a waste collection approach. It takes into consideration the receipt of moving data. Receiving data in movement is very different compared to receiving statically. The paper concludes the study by proposing a guideline for the development of intelligent multiobjective approaches based on algorithms with a weighted sum to convert it into a single goal model through known priorities and loss-measuring capabilities.

Human factors are another critical aspect that should be considered during the design of the solid waste management solution, since smart compartments will be positioned in public areas, such as parks, stadiums, and streets where parties, religious events, music events, sporting events, and widespread demonstrations are usually held. Clusters of people are the most part characterized by the diversity of both physical and socioeconomic as well as cultural characteristics that demonstrate a different behavior when organized in large numbers of people. Studies are applied through simulations in large crowds on different aspects to understand their behavior and establish standards and turn them into heuristics that will help in decision making and predict certain situations, mainly, in evacuation when the so-called panic occurs. In Reference [124], a study is applied through dynamic multisimulation based on modeling agents applied to both urban space and individuals within a crowd. This dynamic 
becomes important within the concept of solid waste management to predict behavior when present in large clusters of people.

Another important aspect that must be considered within a waste management system is the consumption of the battery generated by the use of the devices/sensors available in a solution. The IoT nodes used in solid waste management include ultrasonic sensors and load cell [125], GPS [126], actuators [36], microcontrollers [127], and transmission modules [128]. Some studies describe the use of renewable energy through photovoltaic panels for battery charge and avoid constant manual intervention for replacement. There are also aspects related to the network topology employed in the solution, such as point-to-point model topology, star model, hierarchical model, etc. Depending on the used topology model, nodes can consume more or less energy, just as the distance of node positioning should also be considered. Nodes closer to the base radio tend to spend less energy than nodes located at the periphery of the cell, bearing in mind that transmission modules are responsible for the higher energy consumption. The communication technology employed is also an aspect to be considered, whenever possible, to use low power technologies, such as 6LoWPAN [60], BLE [109], etc., as well as to consider the aspects of sensing, such as whether it will occur periodically, continuously, reactively, or in real time. Based on the aspects mentioned above in Reference [119], a study was directed at the development of a sensor node for the real time monitoring of the level of filling of dumps based on the low energy consumption and the cost containment. They are retained not only to the sensor node more efficiently in terms of energy levels but also to the architecture of the solution, taking in account microcontrollers that can attend the computational capacity with low energy consumption and also to use policies that shut down the node or even part of it when idle.

Another very favorable aspect that can help solid waste management systems is using data analysis to provide the capacity to forecast the generation of waste from a municipality in the short term. Based on data already collected within a specified period, an analysis will be applied, through which more efficient collection planning is enabled and even resources are estimated for a probable growth in the generation of waste that leads to higher investments in infrastructure. Some studies are based on the artificial neural network as presented in Reference [129] that uses input variables related to environmental issues, socioeconomic issues, changes in consumption patterns, and even population growth associated with migration. Through these analyses, it is possible to select the best results based on these performance indices applied for each criterion and thus improve municipal solid waste management implementations. Additionally, in Reference [130], a time series of autoregressive artificial neural networks is used to predict the monthly generation of residue since the characteristics of the solid waste generated are different in different places.

The analysis of the related literature identified open issues that can be addressed in further research work. They can be considered as improvements for proposed systems and identified as follows:

- A waste management platform focusing on citizens' perspective that can interact with the system through a mobile application that, through its location, finds the bins closest to their residence with the respective level of use. Knowing this information, the user can choose to discard the garbage at that moment in a container that has availability or even to retain it and wait until the collection system empties the deposit. In this way, the user will be contributing to the non-overflow of the containers and avoiding that their waste is exposed in the open. The solution includes a physical compartment (bin) equipped with sensors, which performs a continuous sensing of both volume and weight of the residues contained inside. The sensors are managed by an integrated development environment (IDE) microcontroller that also controls communication through a coupled module. The data are transmitted to a middleware, where they are stored and made available to a mobile application [131].

- The waste management system may be the future object of study for solutions of a shorter path for collection routes, that is, trucks already leave with a route previously traced searching for the containers that need emptying. In this way, it is possible to achieve better collection effectiveness in a shorter time and with low fuel consumption. There are many studies available in the literature 
that present different solutions for the shortest path in collection routes. A waste management platform that focuses on citizens' perspective, as above described, coupled with a resolution of the best collection path can bring enormous gains to smart cities.

- The waste management system can be integrated with future parking management studies for vehicle parking. Containers can be positioned as a gateway for parking sensors, which already have an integrated transmission system and the information is presented to users through an application that traces the route from the original position of the vehicle to the available parking space. With a built-in waste management infrastructure, it is possible to add new applications to the base system. A good example would be parking management using infrared presence sensors based on the standard IEEE 802.15.4 with intelligent dumps, which transmit data to the same integrated middleware and, later, are made available to the user through a mobile platform.

- Another point that may be the focus of future work is the deepened study on the life cycle of rechargeable batteries to be used in waste management systems. As these batteries are fixed in the containers that most of the time will be propitious climatic actions, an analysis of the functions of the cell and its interactions with the environment must be studied, as well as its handling and protection against the increase of temperature due to outbreaks due. In this case, the study is not targeted at applications based on smart cities, but the development of new generation batteries can benefit many applications and contribute significantly to the evolution of projects within science and technology.

\section{Lessons Learned}

As lessons learned, some aspects related to the addressed topic can be considered and shared with the community. Using a waste management system alone, does not solve the problem of open garbage accumulation due to excessive discards at inopportune times by citizens, or even the lack of timely collection by competent authorities. People need to raise awareness and exercise citizenship to avoid waste discarding at inappropriate times (for people, the most important thing is to know the location of the nearest ecological point with resources available for that and the closest ecological points for a possible discard). It is also important that concessionaires responsible for public collection always meet the demand in time, changing the current collection systems based on weekly schedules by a methodology based on demand, i.e., mapping the areas with the most significant needs.

Another point observed is related to the final cost of the solution when projected to a municipal scale. To avoid the financial impracticability of the solution, it is necessary to create ways to capitalize resources to be invested directly in solutions to meet the need of municipalities with fewer investments. A good example may be visual advertising inserted directly into smart containers or even through non-invasive advertisements presented in mobile applications integrated in complete waste management solutions.

\section{Conclusions}

To achieve the transformation of traditional cities into smart cities, waste management becomes a critical element in achieving sustainability, efficiency in public spending, improving urban mobility, and preserving natural resources. Recent literature has been revised to investigate variant characteristics and aspects of intelligent waste management systems using the Internet of Things. Since the deployment of IoT infrastructures can enable many opportunities, first, the main search motives were identified, and some useful application models on the topic of waste management were described. Through a detailed literature review, solutions to identified problems were described, considering data detection, transmission, analysis, and processing of collected data, and obtaining the final result for an efficient handling solution for solid waste. Using IoT, it is possible to track the location of waste containers, monitoring the level of garbage deposited, identify locations with the highest demand, suggest the shortest route for collection optimization of solid waste, or even interface with citizens to encourage disposal at times when the container can receive waste, which promotes citizenship and avoids significant problems resulting from the accumulation of garbage outside garbage collectors. 
Author Contributions: K.P. collected and performed a deep analysis and review the related literature on the topic, wrote the first draft of the document, performed the comparison study, and identified some open research issues. J.J.P.C.R. supervised all the study, consolidated the comparison analysis and open issues, and reviewed the structure and the first draft. S.A.K., N.K., and A.F. reviewed the text carefully, verified the comparison study, and reviewed the identified open issues. All the authors contributed equally to the scope definition, motivation, and focus of the paper.

Funding: This work has been supported by national funding from the FCT—Fundação para a Ciência e a Tecnologia through the UID/EEA/50008/2013 Project; by the Government of the Russian Federation, Grant 08-08; by Finep, with resources from Funttel, Grant No. 01.14.0231.00, under the Centro de Referência em Radiocomunicações-CRR project of the Instituto Nacional de Telecomunicações (Inatel), Brazil; by Finatel through the Inatel Smart Campus project; and by the Brazilian National Council for Research and Development (CNPq) via Grant No. 309335/2017-5.

Conflicts of Interest: The authors declare no conflict of interest.

\section{References}

1. MICE Industry by Event Type. Global Opportunity Analysis and Industry Forecast. 2017-2023. Available online: https:/ / www.alliedmarketresearch.com/MICE-industry-market (accessed on 31 May 2018).

2. Krol, E.; Hoffman, E. What is the Internet? Internet Engineering Task Force (IETF): Fremont, CA, USA, 1993. Available online: https:/ / tools.ietf.org/html/rfc1462 (accessed on 31 May 2018).

3. Weber, M.; Lučić, D.; Lovrek, I. Internet of Things context of the smart city. IEEE Conf. Publ. 2017, 187-193. [CrossRef]

4. Zanella, A.; Vangelista, L. Internet of Things for Smart Cities. IEEE Internet Things J. 2014, 1, 22-32. [CrossRef]

5. Attaran, A.; Rashidzadeh, R. Chipless Radio Frequency Identification Tag for IoT Applications. IEEE Internet Things J. 2016, 3, 1310-1318. [CrossRef]

6. Ramnath, S.; Javali, A.; Narang, B.; Mishra, P.; Routray, S.K. IoT based localization and tracking. In Proceedings of the IEEE Conferences, International Conference on IoT and Application (ICIOT), Nagapattinam, India, 19-20 May 2017; pp. 1-4.

7. Tervonen, J.; Mikhaylov, K.; Pieskä, S.; Jämsä, J.; Heikkilä, M. Cognitive Internet-of-Things solutions enabled by wireless sensor and actuator networks. In Proceedings of the IEEE Conferences, 5th IEEE Conference on Cognitive Infocommunications (CogInfoCom), Vietri sul Mare, Italy, 5-7 November 2014; pp. 97-102.

8. Evans, D. The Internet of Things: How the Next Evolution of the Internet is Changing Everything, White Paper; CISCO: San Jose, CA, USA, 2011. Available online: http://www.cisco.com/c/dam/en_us/about/ac79/ docs/innov/IoT_IBSG_0411FINAL.pdf (accessed on 27 November 2018).

9. Campos, L.B.; Cugnasca, C.E.; Hirakawa, A.R.; Martini, J.S.C. Towards an IoT-based system for Smart City. IEEE Conf. 2016, 129-130. [CrossRef]

10. Chuah, J.W. The Internet of Things: An overview and new perspectives in systems design. In Proceedings of the IEEE Conferences, International Symposium on Integrated Circuits (ISIC), Singapore, 10-12 December 2014; pp. 216-219.

11. Atzori, L.; Iera, A.; Morabito, G. The Internet of Things: A survey. Comput. Netw. 2010, 54, 2787-2805. [CrossRef]

12. Pellicer, S.; Santa, G.; Bleda, A.L.; Maestre, R.; Jara, A.J.; Skarmeta, A.G. A Global Perspective of Smart Cities: A Survey. In Proceedings of the International Conference on Innovative Mobile and Internet Services in Ubiquitous Computing, Taichung, Taiwan, 3-5 July 2013; pp. 439-444.

13. Sumi, L.; Ranga, V. Sensor enabled Internet of Things for smart cities. In Proceedings of the IEEE Conferences-Fourth International Conference on Parallel, Distributed and Grid Computing (PDGC), Waknaghat, India, 22-24 December 2016; pp. 295-300.

14. Raaijen, T.; Daneva, M. Depicting the smarter cities of the future: A systematic literature review \& field study. In Proceedings of the Smart City Symposium Prague (SCSP), Prague, Czech Republic, 25-26 May 2017; pp. 1-10.

15. Arasteh, H.; Hosseinnezhad, V.; Loia, V.; Tommasetti, A.; Troisi, O.; Shafie-khah, M.; Siano, P. IoT-based smart cities: A survey. In Proceedings of the IEEE 16th International Conference on Environment and Electrical Engineering (EEEIC), Florence, Italy, 7-10 June 2016; pp. 1-6.

16. Caragliu, A.; Delbo, C.; Nijkamp, P. Smart Cities in Europe. Available online: http://www3.ekf.tuke.sk/ cers/cers2009/PDF/01_03_Nijkamp.pdf (accessed on 31 May 2018). 
17. Wijaya, A.S.; Zainuddin, Z.; Niswar, M. Design a smart waste bin for smart waste management. In Proceedings of the 5th International Conference on Instrumentation, Control, and Automation (ICA), Yogyakarta, Indonesia, 9-11 August 2017; pp. 62-66.

18. Karadimas, D.; Papalambrou, A.; Gialelis, J.; Koubias, S. An integrated node for Smart-City applications based on active RFID tags; Use case on waste-bins. In Proceedings of the 21st International Conference on Emerging Technologies and Factory Automation (ETFA), Berlin, Germany, 6-9 September 2016; pp. 1-7.

19. Al Mamun, M.A.; Hannan, M.A.; Islam, M.S.; Hussain, A.; Basri, H. Integrated sensing and communication technologies for automated solid waste bin monitoring system. In Proceedings of the IEEE Student Conference on Research and Development, Putrajaya, Malaysia, 16-17 December 2013; pp. 480-484.

20. Al Mamun, M.A.; Hannan, M.A.; Hussain, A. Real time solid waste bin monitoring system framework using wireless sensor network. In Proceedings of the International Conference on Electronics, Information and Communications (ICEIC), Kota Kinabalu, Malaysia, 15-18 January 2014; pp. 1-2.

21. Islam, M.S.; Arebey, M.; Hannan, M.A.; Basri, H. Overview for solid waste bin monitoring and collection system. In Proceedings of the International Conference on Innovation Management and Technology Research, Malacca, Malaysia, 21-22 May 2012; pp. 258-262.

22. Choudhary, G.B.; Jain, A.K. Internet of Things: A Survey on Architecture, Technologies, Protocols and Challenges. In Proceedings of the 2016 International Conference on Recent Advances and Innovations in Engineering (ICRAIE), Jaipur, India, 23-25 December 2016; pp. 1-8.

23. Kumar, S.V.; Kumaran, T.S.; Kumar, A.K.; Mathapati, M. Smart garbage monitoring and clearance system using internet of things. In Proceedings of the IEEE International Conference on Smart Technologies and Management for Computing, Communication, Controls, Energy and Materials (ICSTM), Chennai, India, 2-4 August 2017; pp. 184-189.

24. Borozdukhin, A.; Dolinina, O.; Pechenkin, V. Approach to the garbage collection in the "Smart Clean City" project. In Proceedings of the IEEE Conferences, International Colloquium on Information Science and Technology (CiSt), Tangier, Morocco, 24-26 October 2016; pp. 918-922.

25. Sathish, S.; Prabhakaran, M. Conventional solid waste management technique for eradication of solid waste and its impact assessment. In Proceedings of the IEEE Conferences, International Conference on Green technology and environmental Conservation, Chennai, India, 15-17 December 2011; pp. 159-161.

26. Mi, L.; Liu, N.; Zhou, B. Disposal Methods for Municipal Solid Wastes and Its Development Trend. In Proceedings of the 4th International Conference on Bioinformatics and Biomedical Engineering, Chengdu, China, 18-20 June 2010; pp. 1-4.

27. Kawai, K.; Mai Huong, L.T. Key parameters for behaviour related to source separation of household organic waste: A case study in Hanoi, Vietnam. Waste Manag. Res. 2017, 35, 246-252. [CrossRef] [PubMed]

28. Seyring, N.; Dollhofer, M.; Weißenbacher, J.; Bakas, L.; McKinnon, D. Assessment of collection schemes for packaging and other recyclable waste in European Union-28 Member States and capital cities. Waste Manag. Res. 2016, 34, 947-956. [CrossRef] [PubMed]

29. Zobel, T. ISO 14001 adoption and industrial waste generation: The case of Swedish manufacturing firms. Waste Manag. Res. 2015, 33, 107-113. [CrossRef]

30. Ali, M.; Wang, W.; Chaudhry, N.; Geng, Y. Hospital waste management in developing countries: A mini review. Waste Manag. Res. 2017, 35, 581-592. [CrossRef]

31. Bacot, H.; McCoy, B.; Plagman-Galvin, J. Municipal Commercial Recycling Barriers to Success. Am. Rev. Public Adm. 2002, 32, 145-165. [CrossRef]

32. Krzywoszynska, A. Waste? You Mean By-Products! from Bio-Waste Management to Agro-Ecology in Italian Winemaking and beyond. Sociol. Rev. 2012, 60, 47-65. [CrossRef]

33. Babu, B.R.; Parande, A.K.; Basha, C.A. Electrical and electronic waste: A global environmental problem. Waste Manag. Res. 2007, 25, 307-318.

34. Gan, L.; Yang, S. Legal context of high level radioactive waste disposal in China and its further improvement. Energy Environ. 2017, 28, 484-498. [CrossRef]

35. Catania, V.; Ventura, D. An Approch for Monitoring and Smart Planning of Urban Solid Waste Management Using Smart-M3 Platform. In Proceedings of the 15th Conference of Open Innovations Association FRUCT, St. Petersburg, Russia, 21-25 April 2014; pp. 24-31. 
36. Anagnostopoulos, T.; Zaslavsky, A.; Kolomvatsos, K.; Medvedev, A.; Amirian, P.; Morley, J.; Hadjiefthymiades, S. Challenges and Opportunities of Waste Management in IoT-enabled Smart Cities: A Survey. IEEE Trans. Sustain. Comput. 2017, 2, 275-289. [CrossRef]

37. Aazam, M.; St-Hilaire, M.; Lung, C.; Lambadaris, I. Cloud-based smart waste management for smart cities. In Proceedings of the IEEE Workshop on Computer-Aided Modeling and Design of Communication Links (CAMAD 2016), Toronto, ON, Canada, 23-25 October 2016; pp. 188-193.

38. Ramasami, K.; Velumani, B. Location prediction for solid waste management-A Genetic algorithmic approach. In Proceedings of the 2016 IEEE International Conference on Computational Intelligence and Computing Research (ICCIC), Chennai, India, 15-17 December 2016; pp. 1-5.

39. Saha, H.N.; Auddy, S.; Pal, S.; Kumar, S.; Pandey, S.; Singh, R.; Singh, A.K.; Banerjee, S.; Ghosh, D.; Saha, S. Waste management using Internet of Things (IoT). IEEE Conf. Publ. 2017, 359-363. [CrossRef]

40. Chowdhury, B.; Chowdhury, M.U. RFID-based real-time smart waste management system. In Proceedings of the 2007 Australasian Telecommunication Networks and Applications Conference, Christchurch, New Zealand, 2-5 December 2007; pp. 175-180.

41. Lu, J.-W.; Chang, N.-B.; Liao, L.; Liao, M.-Y. Smart and Green Urban Solid Waste Collection Systems: Advances, Challenges, and Perspectives. IEEE Syst. J. 2017, 11, 2804-2817. [CrossRef]

42. Wahab, M.H.A.; Kadir, A.A.; Tomari, M.R.; Jabbar, M.H. Smart Recycle Bin: A Conceptual Approach of Smart Waste Management with Integrated Web Based System. In Proceedings of the 2014 International Conference on IT Convergence and Security (ICITCS), Beijing, China, 28-30 October 2014; pp. 1-4.

43. Thakker, S.; Narayanamoorthi, R. Smart and wireless waste management. In Proceedings of the 2015 International Conference on Innovations in Information, Embedded and Communication Systems (ICIIECS), Coimbatore, India, 19-20 March 2015; pp. 1-4.

44. Folianto, F.; Low, Y.S.; Yeow, W.L. Smartbin: Smart waste management system. In Proceedings of the 2015 IEEE Tenth International Conference on Intelligent Sensors, Sensor Networks and Information Processing (ISSNIP), Singapore, 7-9 April 2015; pp. 1-2.

45. Ramya, E.; Sasikumar, R. A survey of smart environment conservation and protection for waste management. In Proceedings of the 2017 Third International Conference on Advances in Electrical, Electronics, Information, Communication and Bio-Informatics (AEEICB), Chennai, India, 27-28 February 2017; pp. 242-245.

46. Parkash, P.V. IoT Based Waste Management for Smart City. Int. J. Innov. Res. Comput. Commun. Eng. 2016, 4 , 1267-1274.

47. Fallavi, K.N.; Kumar, V.R.; Chaithra, B.M. Smart waste management using Internet of Things: A survey. In Proceedings of the 2017 International Conference on I-SMAC (IoT in Social, Mobile, Analytics and Cloud) (I-SMAC), Palladam, India, 10-11 February 2017; pp. 60-64.

48. Manqele, L.; Adeogun, R.; Dlodlo, M.; Coetzee, L. Multi-objective decision-making framework for effective waste collection in smart cities. In Proceedings of the Global Wireless Summit (GWS), Cape Town, South Africa, 15-18 October 2017; pp. 155-159.

49. Omara, A.; Gulen, D.; Kantarci, B.; Oktug, S. Trajectory Assisted Municipal Agent Mobility: A Sensor-Driven Smart Waste Management System. J. Sens. Actuator Netw. 2018, 7, 29. [CrossRef]

50. Jouhara, H.; Czajczyńska, D.; Ghazal, H.; Krzyzynska, R.; Anguilano, L.; Reynolds, A.; Spencer, N. Municipal waste management systems for domestic use. Energy 2017, 139, 485-506. [CrossRef]

51. Khan, R.; Khan, S.U.; Zaheer, R.; Khan, S. Future Internet: The Internet of Things Architecture, Possible Applications and Key Challenges. In Proceedings of the 2012 10th International Conference on Frontiers of Information Technology, Islamabad, India, 17-19 December 2012; pp. 257-260.

52. Weyrich, M.; Ebert, C. Reference Architecture for the Internet of Things. IEEE J. Mag. 2016, 33, 112-116. [CrossRef]

53. Yang, Z. Study and application on the architecture and keytechnologies for IOT. In Proceedings of the 2011 International Conference on Multimedia Technology, Hangzhou, China, 26-28 July 2011; pp. 747-751.

54. Wu, M.; Lu, T.J.; Ling, F.Y.; Sun, J.; Du, H.Y. Research on the architecture of Internet of Things. In Proceedings of the 2010 3rd International Conference on Advanced Computer Theory and Engineering(ICACTE), Chengdu, China, 20-22 August 2010; pp. V5-484-V5-487.

55. Tan, L.; Wang, N. Future Internet: The Internet of Things. In Proceedings of the 2010 3rd International Conference on Advanced Computer Theory and Engineering (ICACTE), Chengdu, China, 20-22 August 2010; pp. V5-376-V5-380. 
56. Chaqfeh, M.A.; Mohamed, N. Challenges in middleware solutions for the Internet of Things. In Proceedings of the 2012 International Conference on Collaboration Technologies and Systems (CTS), Denver, CO, USA, 21-25 May 2012; pp. 21-26.

57. Al-Fuqaha, A.; Guizani, M.; Mohammadi, M.; Aledhari, M.; Ayyash, M. Internet of Things: A Survey on Enabling Technologies, Protocols, and Applications. IEEE Commun. Surv. Tutor. 2015, 17, 2347-2376. [CrossRef]

58. Koshizuka, N.; Sakamura, K. Ubiquitous ID: Standards for Ubiquitous computing and the Internet of Things. IEEE Pervasive Comput. 2010, 9, 98-101. [CrossRef]

59. Fargas, B.C.; Petersen, M.N. GPS-free geolocation using LoRa in low-power WANs. In Proceedings of the 2017 Global Internet of Things Summit (GIoTS), Geneva, Switzerland, 6-9 June 2017.

60. Kushalnagar, N.; Montenegro, G.; Schumacher, C. IPv6 over Low-Power Wireless Personal Area Networks (6LoWPANs): Overview, Assumptions, Problem Statement, and Goals, RFC4919; Internet Eng. Task Force (IETF): Fremont, CA, USA, 2007; Volume 10.

61. Dhanoriya, S.; Pandey, M. A survey on wireless sensor networks: Faults, misbehaviour and protection against them. In Proceedings of the 8th International Conference on Computing, Communication and Networking Technologies (ICCCNT), Delhi, India, 3-5 July 2017; pp. 1-7.

62. Carvalho, J.C.d.S.; da Rocha, A.R.; Sousa, F.R.C.; Torres, A.B.B.; de Souza, J.N. Management of Multiple Applications for Shared Sensor and Actuators Networks. IEEE J. Mag. 2016, 14, 4358-4366.

63. Ferro, E.; Potorti, F. Bluetooth and Wi-Fi wireless protocols: A survey and a comparison. IEEE Wirel. Commun. 2005, 12, 12-26. [CrossRef]

64. McDermott-Wells, P. What is Bluetooth? IEEE Potentials 2005, 23, 33-35. [CrossRef]

65. Andrei, M.L.; Rădoi, L.A.; Tudose, D.S. Measurement of node mobility for the LoRa protocol. In Proceedings of the 2017 16th RoEduNet Conference: Networking in Education and Research (RoEduNet), Targu Mures, Romania, 21-23 September 2017; pp. 1-6.

66. Crosby, G.V.; Vafa, F. Wireless sensor networks and LTE-A network convergence. In Proceedings of the 38th Annual IEEE Conference on Local Computer Networks, Sydney, NSW, Australia, 21-24 October 2013; pp. 731-734.

67. Ghosh, A.; Ratasuk, R.; Mondal, B.; Mangalvedhe, N.; Thomas, T. LTE-dvanced: Next-generation wireless broadband technology [Invited Paper]. IEEE Wireless Commun. 2010, 17, 10-22. [CrossRef]

68. Riekki, J.; Sanchez, I.; Pyykkonen, M. NFC-Based User Interfaces. In Proceedings of the 2012 4th International Workshop on Near Field Communication, Helsinki, Finland, 13 March 2012; pp. 3-9.

69. Kshetrimayum, R.S. An introduction to UWB communication systems. IEEE Potentials 2009, 28, 9-13. [CrossRef]

70. Xin Ma, X.; Luo, W. The Analysis of 6LowPAN Technology. IEEE Conf. IEEE Pacific-Asia Workshop Comput. Intell. Ind. Appl. 2008, 1, 963-966.

71. Want, R. An introduction to RFID technology. IEEE Pervasive Comput. 2006, 5, 25-33. [CrossRef]

72. Valente, F.F.; Neto, A.C. Intelligent steel inventory tracking with IoT/RFID. In Proceedings of the 2017 IEEE International Conference on RFID Technology \& Application (RFID-TA), Warsaw, Poland, 20-22 September 2017; pp. 158-163.

73. IEEE Standard for Local and Metropolitan Area Networks. Part 15.4: Low-Rate Wireless Personal Area Networks (LR-WPANs); IEEE: New York, NY, USA, 2011; IEEE Std. 802.15.4.

74. Reddy, P.S.N.; Naik, R.N.; Kumar, A.A.; Kishor, S.N. Wireless dust bin monitoring and alert system using Arduino. In Proceedings of the 2017 Second International Conference on Electrical, Computer and Communication Technologies (ICECCT), Coimbatore, India, 22-24 February 2017; pp. 1-5.

75. Sklavos, N.; Zaharakis, I.D.; Kameas, A.; Kalapodi, A. Security \& Trusted Devices in the Context of Internet of Things (IoT). In Proceedings of the IEEE Conferences, Euromicro Conference on Digital System Design (DSD), Vienna, Austria, 30 August-1 September 2017; pp. 502-509.

76. Vinh, T.Q. Real-time traffic sign detection and recognition system based on friendlyARM Tiny 4412 board. In Proceedings of the IEEE Conferences, International Conference on Communications, Management and Telecommunications (ComManTel), DaNang, Vietnam, 28-30 December 2015; pp. 142-146.

77. Jie, L.; Ghayvat, H.; Mukhopadhyay, S.C. Introducing Intel Galileo as a development platform of smart sensor: Evolution, opportunities and challenges. In Proceedings of the IEEE Conferences, 10th Conference on Industrial Electronics and Applications (ICIEA), Auckland, New Zealand, 15-17 June 2015; pp. 1797-1802. 
78. Besari, A.R.A.; Wobowo, I.K.; Sukaridhoto, S.; Setiawan, R.; Rizqullah, M.R. Preliminary design of mobile visual programming apps for Internet of Things applications based on Raspberry Pi 3 platform. In Proceedings of the International Electronics Symposium on Knowledge Creation and Intelligent Computing (IES-KCIC), Surabaya, Indonesia, 26-27 September 2017; pp. 50-54.

79. Hodges, S.; Taylor, S.; Villar, N.; Scott, J.; Bial, D.; Fischer, P.T. Prototyping Connected Devices for the Internet of Things. IEEE J. Mag. 2013, 46, 26-34. [CrossRef]

80. He, N.; Qian, Y.; Huang, H. Experience of teaching embedded systems design with BeagleBone Black board. In Proceedings of the IEEE Conferences, International Conference on Electro Information Technology (EIT), Grand Forks, ND, USA, 19-21 May 2016.

81. Rahmatullah, D.K.; Nasution, S.M.; Azmi, F. Implementation of low interaction web server honeypot using cubieboard. In Proceedings of the International Conference on Control, Electronics, Renewable Energy and Communications (ICCEREC), Bandung, Indonesia, 13-15 September 2016; pp. 127-131.

82. Mian, A.N.; Alvi, S.A.; Khan, R.; Zulqarnain, M.; Iqbal, W. Experimental study of link quality in IEEE 802.15.4 using Z1 Motes. In Proceedings of the International Wireless Communications and Mobile Computing Conference (IWCMC), Paphos, Cyprus, 5-9 September 2016; pp. 830-835.

83. Peterman, D.; James, K.; Glavac, V. Distortion measurement and compensation in a synthetic aperture radar phased-array antenna. In Proceedings of the 14th International Symposium on Antenna Technology and Applied Electromagnetics \& the American Electromagnetics Conference, Ottawa, ON, Canada, 5-8 July 2010; pp. 1-5.

84. Zhong, C.; Eliasson, J.; Makitaavola, H.; Zhang, F. A Cluster-Based Localization Method Using RSSI for Heterogeneous Wireless Sensor Networks. In Proceedings of the 6th International Conference on Wireless Communications Networking and Mobile Computing (WiCOM), Chengdu, China, 23-25 September 2010; pp. 1-6.

85. Bukkapatnam, S.T.S.; Mukkamala, S.; Kunthong, J.; Sarangan, V.; Komanduri, R. Real-time monitoring of container stability loss using wireless vibration sensor tags. In Proceedings of the International Conference on Automation Science and Engineering, Bangalore, India, 22-25 August 2009; pp. 221-226.

86. Levis, P. TinyOS: An Operating System for Sensor Networks, in Ambient Intelligence; Springer: New York, NY, USA, 2005; pp. 115-148.

87. Cao, Q.; Abdelzaher, T.; Stankovic, J.; He, T. The LiteOS operating system: Towards Unix-like abstractions for wireless sensor networks. In Proceedings of the 7th International Conference on Information Processing in Sensor Networks, St. Louis, MO, USA, 22-24 April 2008; pp. 233-244.

88. Baccelli, E.; Hahm, O.; Günes, M.; Wählisch, M.; Schmidt, T.C. RIOT OS: Towards an OS for the Internet of Things. In Proceedings of the 2013 IEEE Conference on Computer Communications Workshops (INFOCOM WKSHPS), Turin, Italy, 14-19 April 2013; pp. 79-80.

89. Dunkels, A.; Gronvall, B.; Voigt, T. Contiki-A lightweight and flexible operating system for tiny networked sensors. In Proceedings of the 29th Annual IEEE International Conference on Local Computer Networks, Tampa, FL, USA, 16-18 November 2004; pp. 455-462.

90. Caputo, D.; Mainetti, L.; Patrono, L.; Vilei, A. Implementation of the EXI Schema on Wireless Sensor Nodes Using Contiki. In Proceedings of the Sixth Internacional Conference on Innovation Mobile and Internet Services in Ubiquitous Computing, Palermo, Italy, 4-6 July 2012; pp. 770-774.

91. Lingyun, Y.; Liging, H.; Manman, Z.; Mingli, Z. RFID data fusion algorithm based on spatio-temporal demantics in Internet of Things. In Proceedings of the 13th IEEE International Conference on Eletronic Measurement \& Instruments (ICEMI), Yangzhou, China, 20-22 October 2017; pp. 179-184.

92. Da Cruz, M.A.A.; Rodrigues, J.J.P.C.; Al-Muhtadi, J.; Korotaev, V.V.; de Albuquerque, V.H.C. A Reference Model for Internet of Things Middleware. IEEE Internet Things J. 2018, 5, 871-883. [CrossRef]

93. Shelby, Z.; Hartke, K.; Bormann, C.; Frank, B. Constrained Application Protocol (CoAP). draft-ietf-core-coap-18; Internet Engineering Task Force (IETF): Fremont, CA, USA, 2013. Available online: https://tools.ietf.org/ html/draft-ietf-core-coap-18 (accessed on 30 June 2018).

94. Bormann, C.; Castellani, A.P.; Shelby, Z. CoAP: An application protocol for billions of tiny Internet nodes. IEEE Internet Comput. 2012, 16, 62-67. [CrossRef]

95. Fielding, R.T. Architectural Styles and the Design of Network-Based Software Architectures. Ph.D. Thesis, University California, Irvine, CA, USA, 2000. 
96. Hunkeler, U.; Truong, H.L.; Stanford-Clark, A. MQTT-S-A publish/subscribe protocol for wireless sensor networks. In Proceedings of the 2008 3rd International Conference on Communication Systems Software and Middleware and Workshops (COMSWARE '08), Bangalore, India, 6-10 January 2008; pp. 791-798.

97. Saint-Andre, P. Extensible Messaging and Presence Protocol (XMPP): Core; Internet Engineering Task Force (IETF): Fremont, CA, USA, 2011; ISSN 2070-1721.

98. Kamiya, T.; Schneider, J. Efficient XML Interchange (EXI) Format 1.0; Recommend. REC-Exi-20110310; World Wide Web Consortium: Cambridge, MA, USA, 2011. Available online: https:/ /www.w3.org/TR/2011/RECexi-20110310/ (accessed on 31 May 2018).

99. Waher, P.; Doi, Y. Efficient XML Interchange (EXI) Format; Std. XEP0322; World Wide Web Consortium: Cambridge, MA, USA, 2013. Available online: https://www.w3.org/TR/2013/PER-exi-20131022/ (accessed on 31 May 2018).

100. OASIS. Advanced Message Queuing Protocol (AMQP) Version 1.0; Advancing Open Standards for the Information Society (OASIS): Burlington, MA, USA, 2012.

101. Esposito, C.; Russo, S.; Di Crescenzo, D. Performance assessment of OMG compliant data distribution middleware. In Proceedings of the 2008 IEEE International Symposium on Parallel and Distributed Processing, Miami, FL, USA, 14-18 April 2008; pp. 1-8.

102. Jara, A.J.; Martinez-Julia, P.; Skarmeta, A. Light-weight multicast DNS and DNS-SD (lmDNS-SD): IPv6-based resource and service discovery for the web of things. In Proceedings of the 2012 Sixth International Conference on Innovative Mobile and Internet Services in Ubiquitous Computing, Palermo, Italy, 4-6 July 2012; pp. 731-738.

103. Krochmal, M.; Cheshire, S. DNS-Based Service Discovery; Request for Comments:6763; Internet Engineering Task Force (IETF): Fremont, CA, USA, 2013.

104. IoT Code Recipes: RPL, mDNS and REST. 8 July 2014. Available online: http://github.com/mehdimo/ IoTCodeRecipes (accessed on 30 June 2018).

105. Winter, T. RPL: IPv6 Routing Protocol for Low-Power and Lossy Networks; Request for Comments: 6550; Internet Engineering Task Force (IETF): Fremont, CA, USA, 2012.

106. Clausen, T.; Herberg, U.; Philipp, M. A critical evaluation of the IPv6 routing protocol for low power and ossy networks (RPL). In Proceedings of the 2011 IEEE 7th International Conference on Wireless and Mobile Computing, Networking and Communications (WiMob), Wuhan, China, 10-12 October 2011; pp. 365-372.

107. Kamma, P.K.; Palla, C.R.; Nelakuditi, U.R.; Yarraboth, R.S. Design and implementation of 6LoWPAN border router. In Proceedings of the Thirteenth International Conference on Wireless and Optical Comunications Networks (WOCN), Hyderabad, India, 21-23 July 2016; pp. 1-5.

108. Palattella, M.R. Standardized protocol stack for the Internet of (important) Things. IEEE Commun. Surv. Tuts. 2013, 15, 1389-1406. [CrossRef]

109. Hui, J.W.; Culler, D.E. Extending IP to low-power, wireless personal area networks. IEEE Internet Comput. 2008, 12, 37-45. [CrossRef]

110. Frank, R.; Bronzi, W.; Castignani, G.; Engel, T. Bluetooth low energy: An alternative technology for VANET applications. In Proceedings of the 2014 11th Annual Conference on Wireless On-demand Network Systems and Services (WONS), Obergurgl, Austria, 2-4 April 2014; pp. 104-107.

111. Decuir, J. Introducing Bluetooth smart: Part 1: A look at both classic and new technologies. IEEE Consum. Electron. Mag. 2014, 3, 12-18. [CrossRef]

112. Jones, E.C.; Chung, C.A. RFID and Auto-ID in Planning and Logistics: A Practical Guide for Military UID Applications; CRC Press: Boca Raton, FL, USA, 2011.

113. Siekkinen, M.; Hiienkari, M.; Nurminen, J.K.; Nieminen, J. How low energy is Bluetooth low energy? Comparative measurements with ZigBee/802.15.4. In Proceedings of the 2012 IEEE Wireless Communications and Networking Conference Workshops (WCNCW), Paris, France, 1 April 2012; pp. 232-237.

114. Pauls, F.; Krone, S.; Nitzold, W.; Fettweis, G.; Flores, C. Evaluation of Efficient Modes of Operation of GSM/GPRS Modules for M2M Communications. In Proceedings of the IEEE 78th Vehicular Technology Conference (VTC Fall), Las Vegas, NV, USA, 2-5 September 2013; pp. 1-6.

115. Liu, L.; Liang, Y. A New Architecture Design for WCDMA and GSM Dual-mode Mobile Phones. In Proceedings of the International Conference on Computer Science and Electronics Engineering, Hangzhou, China, 23-25 March 2012; pp. 157-160.

116. Minoli, D. Building the Internet of Things With IPv6 and MIPv6: The Evolving World of M2M Communications; Wiley: New York, NY, USA, 2013. 
117. Singh, G.K.; Gupta, K.; Chaudhary, S. Solid Waste Management: Its Sources, Collection, Transportation and Recycling. Int. J. Environ. Sci. Dev. 2014, 5, 347. [CrossRef]

118. Manliguez, C.; Cuabo, P.; Gamot, R.M.; Ligue, K.D. Solid Waste Collection Routing optimization using Hybridized Modified Discrete Firefly Algorithm and Simulated Annealing, A Case Study in Davao City, Philippines. In Proceedings of the 3rd International Conference on Geographical Information Systems Theory, Applications and Management, Porto, Portugal, 27-28 April 2017; pp. 50-61.

119. Cerchecci, M.; Luti, F.; Mecocci, A.; Parrino, S.; Peruzzi, G.; Pozzebon, A. A Low Power IoT Sensor Node Architecture for Waste Management Within Smart Cities Context. Sensors 2018, 18, 1282. [CrossRef] [PubMed]

120. Jia, D.; Tong, Y.; Yu, Y.; Cai, Z.; Gao, S. A Novel Backtracking Search with Grey Wolf Algorithm for Optimization. In Proceedings of the 10th International Conference on Intelligent Human-Machine Systems and Cybernetics (IHMSC), Hangzhou, China, 25-26 August 2018; Volume 1, pp. 73-76.

121. Yang, M.; Liu, T.; Wang, X.; Yan, Y.; Hu, R.; Zhu, Q. Design of WebGIS System Based on JavaScript and ArcGIS Server. In Proceedings of the International Conference on Smart Grid and Electrical Automation (ICSGEA), Changsha, China, 27-28 May 2017; pp. 709-712.

122. Yiu, Y.F.; Du, J.; Mahapatra, R. Evolutionary Heuristic A* Search: Heuristic Function Optimization via Genetic Algorithm. In Proceedings of the IEEE First International Conference on Artificial Intelligence and Knowledge Engineering (AIKE), Laguna Hills, CA, USA, 26-28 September 2018; pp. 25-32.

123. Gao, Y. An Improved Hybrid Group Intelligent Algorithm Based on Artificial Bee Colony and Particle Swarm Optimization. In Proceedings of the International Conference on Virtual Reality and Intelligent Systems (ICVRIS), Changsha, China, 10-11 August 2018; pp. 160-163.

124. Ochoa, A.; Rudomin, I.; Vargas-Solar, G.; Espinosa-Oviedo, J.A.; Pérez, H.; Zechinelli-Martini, J.L. Humanitarian Logistics and Cultural Diversity within Crowd Simulation. Computación y Sistemas 2017, 21, 7-21.

125. Mohan, A.; Johar, S.; Mini, S. A Waste Collection Mechanism based on IoT. In Proceedings of the 14th IEEE India Council International Conference (INDICON), Roorkee, India, 15-17 December 2017; pp. 1-5.

126. Zeeshan, S.; Shahid, Z.; Khan, S.; Shaikh, F.A. Solid Waste Management in Korangi District of Karachi using GPS and GIS: A Case study. In Proceedings of the 7th International Conference on Computer and Communication Engineering (ICCCE), Kuala Lumpur, Malaysia, 19-20 September 2018; pp. 1-4.

127. Balamurugan, S.; Ajithx, A.; Ratnakaran, S.; Balaji, S.; Marimuthu, R. Design of smart waste management system. In Proceedings of the International Conference on Microelectronic Devices, Circuits and Systems (ICMDCS), Vellore, India, 10-12 August 2017; pp. 1-4.

128. Beliatis, M.J.; Mansour, H.; Nagy, S.; Aagaard, A.; Presser, M. Digital waste management using LoRa network a business case from lab to fab. In Proceedings of the Global Internet of Things Summit (GIoTS), Bilbao, Spain, 4-7 June 2018; pp. 1-6.

129. Estrada, E.; Maciel, R.; Zezzatti, C.A.; Bernabe-Loranca, B.; Oliva, D.; Larios, V. Smart City Visualization Tool for the Open Data Georeferenced Analysis Utilizing Machine Learning. Int. J. Comb. Optim. Probl. Inf. 2018, 9, 25-40.

130. Singh, D.; Satija, A. Prediction of municipal solid waste generation for optimum planning and management with artificial neural network-Case study: Faridabad City in Haryana State (India). Int. J. Syst. Assurance Eng. Manag. 2018, 9, 91-97. [CrossRef]

131. Pardini, K.; Rodrigues, J.J.P.C.; Hassan, S.A.; Kumar, N.; Furtado, V. Smart Waste Bin: A New Approach for Waste Management in Large Urban Centers. In Proceedings of the 3rd International Workshop of Communication for Networked Smart Cities (CorNer 2018), IEEE 88th Vehicular Technology Conference (VTC 2018-Fall), Chicago, IL, USA, 27-30 August 2018.

(C) 2019 by the authors. Licensee MDPI, Basel, Switzerland. This article is an open access article distributed under the terms and conditions of the Creative Commons Attribution (CC BY) license (http:// creativecommons.org/licenses/by/4.0/). 